\section{Safety of Pentavalent Vaccines}

I have been very closely following the various viewpoints in the scientific journals and reports coming out in the media regarding the deaths allegedly reported following introduction of pentavalent vaccine in the states of Kerala and Tamil Nadu, since December 2011.

Currently, pentavalent vaccines are manufactured worldwide by 8 different manufacturers. In addition, five of these vaccines are prequalified by WHO [1]. The prequalification happens only after release of the vaccine by independent international laboratories.

Controversies surrounding the vaccine from Bhutan, and Sri Lanka, are often cited. The pentavalent vaccine was suspended in Bhutan on 23 October 2009, after reports of deaths. However, the investigation by $\mathrm{MOH}$ and WHO concluded that the deaths were coincidental. The vaccine was reintroduced in the country in June 2011 and is used as of now without any adverse reports [2]. Similarly, in Sri Lanka, use was suspended due to reports of a few deaths. The MOH and $\mathrm{WHO}$ found no evidence of a causal relationship. In fact, similar deaths temporally associated with other vaccines continued to be reported even after pentavalent vaccination was temporarily suspended. The vaccine was reintroduced in February 2010 and continues to be used [3].

Vaccines are normally scheduled early in life, when infections and other illnesses are common. It is therefore possible for many Serious Adverse Events Following Immunization (AEFI), including deaths, to be falsely attributed to vaccine through chance association.

The decision to continue inclusion of Hib vaccines in Universal Immunization Programme as liquid Pentavalent vaccine in Kerala and Tamil Nadu and later in six additional states was taken during the $8^{\text {th }}$ meeting of the Mission steering Group of the National Rural Health Mission (NRHM) chaired by the Union Health Minister of Health and Family Welfare. This meeting was attended by Principal Secretaries (Health) of various states, Public Health Professionals, Physicians, Surgeons and senior officials from various Ministries including Ministers of HRD, Rural Development and Women and Child Development. [4]

The Indian Academy of Pediatrics Committee on Immunization (IAPCoI) under my chairmanship first recommended the use of DTPw-HB-Hib pentavalent vaccine for its members in the year 2001 and requested the Government of India (GoI) to include the same in the National Immunization Program. Since then, several thousand Pediatricians and other practitioners all over the country have been using this combination vaccine manufactured by Glaxo Smithkline, Sanofi Aventis and now by indigenous vaccine manufacturers.

The GoI AEFI investigation committee and the Kerala \& TN AEFI investigation committees have ruled out any causal relationship of pentavalent vaccine administration with the deaths reported, establishing causes like other co-morbid conditions in the vaccinees.

The vaccine has been demonstrated to be efficacious and effective in numerous studies and is widely supported by global and Indian health communities.

We should welcome this positive step taken by the Ministry of Health by including the pentavalent vaccine in the Universal Immunization Programme (UIP) in India. Based on the available data, I reiterate that the pentavalent combination vaccines (DTPw-HB-Hib) are very safe and there should not be cause for concern. Moreover, each batch of these vaccines is released by the National Control Laboratory, CDL, Kasauli (H.P.) before it comes to market.

As of October 2012 data, 114 countries in the world have introduced pentavalent vaccine in their national immunization programs [5].

A Parthasarathy National President, Indian Academy of Pediatrics 1997, apartha2020@gmail.com

\section{REFERENCES}

1. WHO prequalified vaccines. Available from: URL:http:// www.who.int/immunization_standards/vaccine_quality/ PQ_vaccine_list_en/en/ Accessed on 21 January, 2013.

2. Pentavalent Vaccine to be reintroduced in June. Bhutan Observer. Available from: http://www.bhutanobserver.bt/ pentavalent-vaccine-to-be-re-introduced-in-june. Accessed on 21 January, 2013.

3. GACVS statement on Pentavalent vaccine use in Asia. Available from: URL: http://www.who.int/vaccine_safety/ pentavalent3.pdf. Accessed on 10 September 2013.

4. Press Information Bureau. Govt. of India, Ministry of Health and family Welfare. 17-April, 2012.

5. WHO vaccine-preventable diseases: monitoring system 2012 global summary Available from: URL: http:// apps.who.int/immunization_monitoring/en/ globalsummary/ScheduleResult.cfm. Accessed on 21 January, 2013. 